\title{
Immediate and delayed contact hypersensitivity to thiocolchicoside
}

\author{
Giuseppe Mancuso ${ }^{1 凶}$
}

\begin{abstract}
Thiocolchicoside is a muscle relaxant, anti-inflammatory, and analgesic. Administered orally, intramuscularly, or topically, this drug is used in the symptomatic treatment of muscular spasms and rheumatologic disorders. Despite its extensive use, thiocolchicoside is a very rare sensitizer. The case reported here is one of simultaneous contact urticaria and a delayed-type contact allergy to thiocolchicoside. The diagnosis was suggested by the patient's history and demonstrated by immediate and delayed positive skin test responses. The results of patch tests on intact skin (negative in immediate reading) and open tests (positive only on inflamed skin) may indicate that epicutaneously applied thiocolchicoside can induce an immediate reaction only when a damaged epidermal barrier is present. In contrast, skin integrity does not seem to prevent the appearance of a delayed-type reaction to this drug.
\end{abstract}

Keywords: thiocolchicoside, immediate and delayed contact hypersensitivity, skin integrity

Received: 14 January 2018 | Returned for modification: 18 April 2018 | Accepted: 7 May 2018

\section{Introduction}

Thiocolchicoside (TCD) is a sulfureted semisynthetic drug derived from colchicoside, a natural glucoside present in the plant Gloriosa superba. Administered orally, parenterally, or topically, the drug is a muscle relaxant, anti-inflammatory, and analgesic commonly used to treat rheumatologic and orthopedic disorders.

Despite the extensive use of TCD, very few cases of documented hypersensitivity reactions have been reported. Clinical presentations of TCD hypersensitivity include photocontact allergy (1), allergic contact dermatitis $(2,3)$, and IgE immediate systemic reaction (4). The a, $\beta$-polyunsaturated ketone group (Fig. 1) is identifiable as allergenic $(2,5)$.

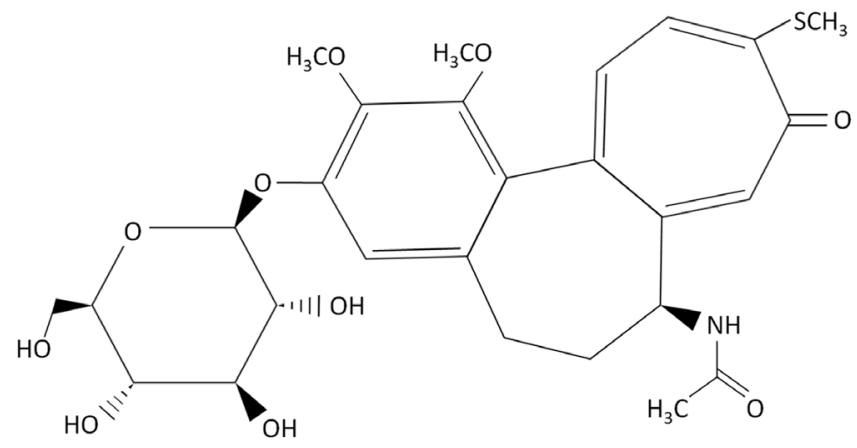

Figure 1 | Chemical structure of thiocolchicoside (2-demethoxy-2-glucosidoxythiocolchicine).

\section{Case report}

A 27-year-old non-atopic woman reported that, 2 months earlier, she had developed an itching eruption immediately after the first application of a cream containing $0.25 \%$ TCD for sciatica pain relief. The lesions mainly involved the right thigh, where the dermatitis had first started. Treatment with topical and systemic corticosteroids led to clearance of the dermatitis in a few days. The patient had previously used the same cream without any reported adverse reactions. A complete personal history documented that the patient suffered from a nickel contact allergy.
She was referred for allergy testing, which was performed under strict medical monitoring and with the consent of the patient.

Initial open tests were performed with the cream (as is) on normal back skin and on previously affected but clinically normal thigh skin. For this purpose, $0.1 \mathrm{ml}$ of the cream was applied over areas measuring $3 \times 3 \mathrm{~cm}$ of both skin regions. No reactions were observed in either skin area at 20, 40, and 60 minutes.

Prick tests on the volar aspects of the forearms were done with the cream (as is), TCD $0.25 \%$ aq., and excipients. These include (with test concentrations and vehicles noted): paraben mix (16\% petrolatum (pet.)), hydrogenated lanolin (30\% pet.), lavender extract ( $5 \%$ pet.), silicone oil ( $5 \%$ pet.), sodium lauryl sulfate $(0.25 \%$ aq.), spermaceti ( $5 \%$ pet.), sodium alginate ( $0.5 \%$ pet.), stearic acid (5\% pet.), and polysorbate 80 (5\% pet.). All of the allergens were prepared in our laboratory except for the paraben mix and polysorbate 80, which were obtained from Chemotechnique Diagnostics AB, Sweden. A positive skin prick test was defined as a wheal $\geq 3 \mathrm{~mm}$ in diameter with surrounding flare that develops 20 minutes after skin testing. Immediate contact hypersensitivity reactions developed to the cream (wheal $7 \times 6 \mathrm{~mm}$, flare $20 \times 25 \mathrm{~mm}$ ) and to TCD $0.25 \%$ aq. (wheal $6 \times 6 \mathrm{~mm}$, flare $15 \times 15 \mathrm{~mm}$; Table 1 ). The wheals persisted for approximately 30 to 60 minutes but disappeared without apparent sequela in less than 2 hours. However, about 2 days later, the patient noticed an eczematous reaction at the sites of the two positive prick tests that persisted for a few days. A negative control (saline) showed no response. Prick tests with TCD $0.25 \%$ aq. were made on seven healthy volunteers and all were negative at 20 minutes, thus excluding an irritative reaction.

Patch tests on intact back skin with the cream and its components showed delayed-type reactions to the cream (as is) and to TCD $1 \%$ aq. at day 2 and day 4 (Table 1). The substances were applied to clinically normal upper back skin using Van der Bend square chambers. The patch test reactions were scored according to the guidelines of the International Contact Dermatitis Research Group. No immediate-type reactions were observed at 20 minutes except for a doubtful reaction (faint erythema without edema) to the cream. Patch tests (reading at day 3) to the European Baseline Series (Chemotechnique Diagnostics) showed a positive reaction $(++)$ to nickel sulfate. Neither immediate nor delayed reactions 
were observed in 11 control subjects patch tested with TCD 1\% aq.

Immediate reactivity to epicutaneously applied TCD was also evaluated on a slightly eczematous area of the patient's chest that had appeared about 20 days earlier due to contact with a necklace of unspecified material. Open tests on the inflamed skin showed a localized urticarial reaction (20 minutes) to the cream (as is) and to TCD $0.25 \%$ aq., along with a negative response to the vehicle of the allergen solution (water) as the negative control. Open tests on the intact chest skin with the same allergens were negative (Table 1).

The patient was unable to say whether inflammatory or traumatic lesions that could have favored permeation of the drug had already been present when she applied the cream.

The patient subsequently avoided contact with TCD-containing products and no symptoms recurred.

Table 1 | Results of skin tests.

\begin{tabular}{lccc}
\hline Test & 20 min & D2 & D4 \\
\hline Prick tests & & & \\
The cream (as is) & + & ER & NT \\
TCD 0.25\% aq. & + & ER & NT \\
Other components of the cream & - & neg. & NT \\
Saline & - & & \\
Histamine 0.1\% saline & + & & \\
Patch tests & & & ++ \\
The cream (as is) & DR & + & - \\
TCD 0.25\% aq. & - & $?+$ & ++ \\
TCD $1 \%$ aq. & - & + & - \\
Other components of the cream & - & - & \\
Open tests & & & NT \\
On normal skin & & & NT \\
The cream (as is) & - & NT & \\
$\quad$ TCD 0.25\% aq. & - & NT & NT \\
On inflamed skin & & & NT \\
The cream (as is) & & NT & NT \\
$\quad$ TCD 0.25\% aq. & + & NT & Negative control (water) \\
\hline
\end{tabular}

$\mathrm{D}=$ day; $\min =$ minutes; $\mathrm{TCD}=$ thiocolchicoside; $N T=$ not tested; $E R=$ eczematous reaction; $\mathrm{DR}=$ doubtful reaction (redness without edema).

\section{Discussion}

The patient's medical history and positivity to prick and patch tests with negative controls suggests the coexistence of immediate and delayed contact hypersensitivity to TCD.

To our knowledge, there are no literature data on documented cases of immediate contact hypersensitivity to TCD. The IgE immediate reaction to TCD described by Caimmi et al. was induced by systemic administration of the drug (4).

In the case presented here, the results of patch tests on intact skin (negative in immediate reading) and open tests (positive only on inflamed skin) may indicate that epicutaneously applied TCD can induce an immediate reaction only when a damaged epidermal barrier is present. This finding is not surprising, considering the physicochemical properties of TCD-in particular its relatively high molecular weight of 563 daltons-which are not favorable to the drug permeating easily and rapidly through skin, as shown by Aguzzi et al. on human excised full-thickness skin specimens (6). In the study by Aguzzi et al. (6), in fact, TCD cutaneous amounts were very low or almost absent within the first hour of incubating the skin samples with TCD-containing products, including a $0.25 \%$ drug aqueous solution. Higher drug quantities were found after increasing the incubation time by using the same simple aqueous solution utilized for skin tests in the case presented here. The clinical transposition of these experimental data could, in the current case, explain the negativity of the immediate skin test (reading within the first hour) as due to an insufficient amount of allergen penetrating into the intact skin. In contrast, a greater cutaneous accumulation of the allergen in the hours following the start of the test would have led to the delayed skin test reaction.

The current case underlines the importance of percutaneous testing (prick test) because routine patch testing and open testing on intact skin would have missed the immediate hypersensitivity to TCD. In the previous studies on contact $(2,3)$ and photocontact allergy (1) to TCD, immediate skin tests were not performed.

Only two cases of allergic contact dermatitis induced by TCD have been reported in the literature $(2,3)$. In those cases, no manifestations that could be attributed to immediate contact hypersensitivity were present. Similar to what was observed in the case presented here, skin integrity does not seem to prevent the appearance of a delayed reaction to an epicutaneously applied drug. In both cases, conventional patch tests with delayed readings gave a positive reaction to TCD on normal back skin $(2,3)$. Furthermore, the occupational allergic contact dermatitis from TCD described by us in one of the two above mentioned cases also appeared on apparently normal skin (3). There, a subsequent flare up of the dermatitis occurred concurrently with the positive patch test reactions to TCD $0.50 \%, 1 \%, 2 \%$, and $5 \%$ pet. A positive reaction (+) to TCD $0.25 \%$ was observed only at day 5 and day 7 .

In their study, Foti et al. believed that $2 \%$ pet. may be adequate for patch testing with TCD (2). In this study, the patch test with TCD was positive at $1 \%$ in an aqueous solution. Due to the solubility characteristics of the drug, water instead of petrolatum was chosen as a vehicle (6).

A literature review shows that many compounds can produce combined eczematous and urticarial reactions with allergic and non-allergic mechanisms. With regard to the latter, examples include benzoic acid, sorbic acid, cinnamaldehyde, and balsam of Peru (among others); these induce non-immunological contact urticaria in many individuals and a delayed reaction in some patients $(7,8)$.

An associated immediate and delayed contact allergy to chlorocresol, chlorhexidine, formaldehyde resin, fragrances, paraphenylenediamine, epoxy resin, penicillin, and protein has also been reported, as it has to caterpillar bite, fish, and chicken meat, among others.

Fragrances, in particular, commonly cause both delayed and immediate allergic patch test reactions, and patients with immediate contact reactions have an increase in delayed contact reactions to the same allergen. In 112 patients with immediate patch test reactions to fragrance mix, and in 113 with similar reactions to balsam of Peru, Katsarou et al. observed associated delayed patch test reactions in $13.4 \%$ and $8.8 \%$ of the patients, respectively (8).

Bergqvist-Karlsson reported a case of both immediate and delayed contact hypersensitivity to chlorhexidine in the same patient. That case is similar to the current case in the way the immediate open test reactions to chlorhexidine occurred. Bergqvist-Karlsson observed these reactions on inflamed skin and not on intact skin, unlike what was observed for delayed patch test reactions occurring on normal skin (9).

In conclusion, TCD should be added to the list of potential agents responsible for immediate and delayed allergic contact reactions, sometimes, as in the case presented here, both in the same subject.

\section{Acknowledgements}

The author thanks Alessandra Mancuso for critically reading the manuscript. 


\section{References}

1. Foti C, Vena GA, Angelini G. Photocontact allergy due to thiocolchicoside. Contact Dermatitis. 1992;27:201-2.

2. Foti C, Cassano N, Mazzarella F, Bonamonte D, Vena GA. Contact allergy to thiocolchicoside. Contact Dermatitis. 1997;37:134.

3. Mancuso G, Berdondini RM. Occupational allergic contact dermatitis from thiocolchicoside. Contact Dermatitis. 2000;43:180-1.

4. Caimmi D, Caviglioli S, Raschetti R, Demoly P. An IgE immediate reaction to thiocolchicoside. Int J Immunopathol Pharmacol. 2012;25:267-8.

5. Giménez-Arnau E. Bio-guided fractionation and identification of allergens in complex mixtures and products. In: Johansen JD, Frosch PJ, Lepoittevin JP, editors. Contact Dermatitis. 5th ed. Springer-Verlag. 2011:111-20.
6. Aguzzi C, Rossi S, Bagnasco M, Lanata L, Sandri G, Bona F, et al. Penetration and distribution of thiocolchicoside through human skin: comparison between a commercial foam (Miotens ${ }^{\circledR}$ ) and a drug solution. AAPS PharmSciTech. 2008;9:1185-90.

7. Rietschel RL, Fowler JF. Contact urticaria. In: Rietschel RL, Fowler JF, editors. Fisher's contact dermatitis. 6th ed. Hamilton, Ontario: BC Decker Inc. 2008:632.

8. Katsarou A, Armenaka M, Kalogeromitros D, Koufou V, Georgala S. Contact reactions to fragrances. Ann Allergy Asthma Immunol. 1999;82:449-55.

9. Bergqvist-Karlsson A. Delayed and immediate-type hypersensitivity to chlorhexidine. Contact Dermatitis. 1988;18:84-8. 